\title{
Differences in running performance of single- and group-housed mice
}

Department of Biomedical Engineering, Johns Hopkins University School of Medicine,

Baltimore, MD, USA

Running Title: $\quad$ Social aspects of voluntary wheel running in mice acquisition.

Correspondence: Patrick O. Kanold, Ph.D., Johns Hopkins University School of Medicine,

Competing Interests: The authors declare no competing interests.

Acknowledgements: POK designed and supervised the study. UP contributed to system

21 design, system construction, and study. UP and POK analyzed data. UP and POK wrote

22 manuscript. We thank Dr. Peter Jendrichovsky and Amber Tietgens for help during data

23 acquisition. Supported by NIH U19NS107474. 


\section{Abstract}

27 Mice are one of several common animal models in neuroscience and mouse behavior is

28 becoming increasingly relevant. Mice are housed either in groups or alone in standard cages

29 during which they show a variety of different behaviors. Moreover, housing conditions might

30 alter the behavior of the mice as do social interactions. When given access to running wheels as

31 an environmental enrichment, mice tend to run long distances preferentially during their dark

32 cycle. However, it is currently not well understood whether and how mice utilize running wheels

33 when single-housed or group-housed. Here we developed a low-cost running wheel data

34 acquisition system to study running in adult C57BL/6 mice at high temporal resolution under

35 different social conditions. As expected, adult C57BL/6 mice prefer to run in stretches during the

36 dark cycle and mostly rest during the light cycle. When single-housed, running bouts occur

37 independent from each other as indicated by an exponential decaying autocorrelation. In

38 contrast, mice run $\sim 50 \%$ less when housed in groups of $n=3$ and their temporal pattern of

39 running exhibits a power law decay in the autocorrelation indicative of potential social

40 interactions. Our results demonstrate that running wheels are a limited resource for which mice

41 compete for when they are group-housed, thereby reducing their overall running activity.

42

43 Significance Statement

44 Voluntary cage wheel running is an important environmental enrichment for mice housed either

45 alone or in groups. We show that this resource is considerably restricted for group-housed mice. 
Introduction

47 Mice are one of several common animal models in neuroscience and the current interest in understanding neural circuitry underlying specific behaviors makes understanding mouse behavior increasingly relevant. In experimental settings, mice are housed either in groups or alone in cages during which mice display a variety of different behaviors. Studies using

51 environmental enrichment have shown a large influence of the housing conditions on the brain

52 (Baroncelli et al., 2010; Kempermann, 2019; Stryker and Löwel, 2018)). Thus, housing conditions as well as social interactions might alter the behavior of mice. One of the simplest behaviors to observe and that is shared between mice and humans is locomotion. possibly social interaction. Locomotion activity patterns are linked to the activation of specific brain regions that are involved in the motivation to run and in controlling the amount of locomotor behavior performed (Rhodes et al., 2003). Mice are nocturnal, demonstrating a nocturnal circadian rhythm in locomotor activity. That is, they run $\sim 5 x$ more during dark compared to the day (Fuochi et al., 2021; Kobayashi et al., 2020). The onset in running after

61 dark is rapid, within $1 \mathrm{hr}$ (Bains et al., 2018; Fuochi et al., 2021; Kopp, 2001). Observations in

62 multiple strains of mice have shown that mice run in intermittent bouts of locomotor activity

63 during the day (Fuochi et al., 2021; Kobayashi et al., 2020).

While bouts of locomotion are present in all mice, locomotion is enhanced when mice have access to a running wheel. In home cages without access to cage wheels, mice exhibit peak running speeds of $\sim 1 \mathrm{~cm} / \mathrm{s}$ covering total distances of $\sim 50 \mathrm{~m}$ during the day and $\sim 150-$

$67200 \mathrm{~m}$ during the $12 \mathrm{~h}$ night cycle (lannello, 2019). The overall locomotion activity changes

68 drastically with access to wheels. In this case, mice show a more distinct night/day activity

69 profile compared to when they don't have access to a wheel, while maintaining some

70 intermittency in activity periods throughout the light/dark (LD)-cycle (Bains et al., 2018). 
71 Moreover, mice run up to $100 x$ longer distances of about $10-20 \mathrm{~km} /$ day with access to a wheel

72 without significant increase in food consumption (Koteja et al., 1999) or major change in body

73 weight (Zhu et al., 2021) demonstrating that running is a higly rewarding activity over alternative

74 environmental enrichments (Sherwin, 1998) at low metabolic cost.

75 This physiological regulation of running in mice has genetic components. Inbred mice

76 remain their circadian rhythm in cage wheel activity (Kopp, 2001) and running can be enhanced

77 through breeding with females running about 3 times more distance than males (12 km vs. 4

78 km/day; (Koteja et al., 1999). Running distance varies in individual mice over days (Goh and

79 Ladiges, 2015) and in general varies by age and strain (Goh and Ladiges, 2015). Running

80 activity in C57BL/6 mice is similar for $4-12$ months of age, but greatly reduced for mice $>20$

81 months of age (Elias et al., 1975; Goh and Ladiges, 2015). Female mice tend to run longer

82 distances than male mice, although in BL/6 strains a reverse trend has been reported with

83 females running $\sim 15 \%$ less that males (Fuochi et al., 2021).

Running is also regulated by neuromodulators. For example dopamine release is

thought to be optimized in high-running rats as both increase/or decrease of $D_{1}$-receptor

stimulation reduces running (Roberts et al., 2012). The site of dopamine action seems to be the

nucleus accumbens where both the $D_{1}$ and $D_{2}$-dopamine receptor stimulation selectively

control wheel running and food intake (Zhu et al., 2016). Specifically, $D_{1}$-dopamine receptor

stimulation increases locomotion, whereas $D_{2}$-dopamine receptor stimulations reduces

locomotion. Dopamine release is also affected by social housing conditions (Pasquarelli et al.,

91 2017), thus the housing conditions of mice might influence wheel running behavior.

Here we studied wheel running in mice during single and group housing. We show group

93 housing reduces overall running activity in group-housed mice by $\sim 50 \%$ compared to single

94 housing and changes their temporal organization in running. Our data suggest that running

95 wheels provide a significant environmental enrichment of which mice compete for when housed

96 in groups. 


\section{Materials and Methods}

99 All animal procedures were approved by the Johns Hopkins University and NIH Animal Care

100 and Use Committee. We used male and female adult mice (C57BL/6J background, Jackson

101 Labs, \#000664).

102 We fitted round, multi-use neodymium permanent magnets $(6 \times 3 \mathrm{~mm}$; e.g. Diymag) to a running

103 wheel (Tecniplast, USA). A magnetic induction switch (Reed; contact normally open; plastic;

104 Wowoone; $2.5 \mathrm{~mm} \times 14 \mathrm{~mm}$ ) was positioned at the back of the wheel. Sensor data was acquired

105 with a National Instruments data acquisition board (NI USB-6215, bus-powered M-series). Our

106 magnetic sensor was stabilized with shrink tubing that neatly fit into the $1 \mathrm{ml}$ tube of a syringe.

107 This arrangement allowed for quick removal of the sensors during cage changes and for wheel

108 washing/sterilization procedures between experiments. We also added an LED that conveyed

109 the activity within each channel without the need for a computer to aid with proper positioning of

110 the magnetic switch prior to recording. The cost for parts beyond the $\mathrm{NI}$-card was $<\$ 10$ per

111 cage. Data was acquired at a $100 \mathrm{~Hz}$ sampling rate using custom scripts in MATLAB

112 (Mathworks).

114 To accustom mice to wheel running before recording, mice were group-housed and separated

115 by sex for up to several weeks with access to a single wheel in each cage. The day of the

116 individual locomotion assessment, animals were single-housed for up to $72 \mathrm{hrs}$ with non-

117 competitive access to a cage wheel attached to the data acquisition system. Locomotion activity

118 was continuously recorded at a sampling rate of $100 \mathrm{~Hz} /$ cage wheel for up $72 \mathrm{hr}$ under a

119 12h:12h light-dark (LD) cycle. Mice were not sound isolated from each other and were free to

120 communicate across cages. Group running was assessed in their respective home cages. 
122 Running activity was analyzed with custom-written code in MATLAB (Mathworks). All data is

123 expressed as mean \pm standard deviation, if not stated otherwise.

125 The autocorrelation was calculated from running activity during the D-phases using a standard

126 z-normalized co-variance measure for a maximal shift of up to $500 \mathrm{~s}$ corrected for tapering.

127 The reduction $(R)$ in wheel running activity during group housing was estimated as the ratio of

$128 R=\left(D_{G r p}-3 \cdot D_{S g l}\right) / 3 \cdot D_{S g l}$, in which $D_{G r p}$ is the mean distance run during group housing. $D_{S g l}$ is

129 the mean distance run of the corresponding 3 mice when single-housed. The ratio was

130 expressed as percentage, i.e. multiplied by 100.

132 Results

133 We studied voluntary wheel running of wildtype C57BI/6 mice in two cohorts, each consisting of

1343 females and 3 males housed in groups (age $3-5$ months; weight $25-35 \mathrm{~g}$ ). In order to study

135 running with high temporal resolution we developed a low-cost system to monitor the running

136 wheels. We instrumented a commercial cage wheel with 4 magnets and a reed switch and

137 aquired signals at $100 \mathrm{~Hz}$ using a data acquisition board (DAQ) board (Fig. 1). Each AD card

138 has 16 channels, allowing us to monitor up to 16 cages. To estimate the running behavior for

139 individual mice, mice were single housed with exclusive wheel access for $2-3$ days followed by

140 measurement of running activity after return to their respective home cages for several days

141 (group housing). For data analysis, measures were averaged over several days of recordings

142 for single-housed and group house conditions. Single-housed running activity occurred in 'bouts'

143 that are abrupt onsets of running activity often at maximal speed that cease equally rapidly (Fig.

$1442 \mathrm{2}, \mathrm{B})$. As expected for nocturnal animals, bouts occurred mostly during the dark phase, could

145 last many hundreds of seconds, and revealed running speeds of up to $\sim 120 \mathrm{~cm} / \mathrm{s}$ resulting in

146 clear peak-velocities in the speed distribution (Fig. 2C, 3A). 
We next assessed the temporal organization of bouts by calculating the autocorrelation

148 for the time course in running activity. If bouts exhibit a specific duration and recur at regular

149 intervals, the autocorrelation function would be oscillatory. Alternatively, if bouts occur randomly

150 with random durations, the autocorrelation decays like an exponential function over time. We

151 found the autocorrelation to decay exponentially, suggesting that bouts during single housing

152 are random occurrence of wheel running (Fig. 2D, 3B).

153 We next measured the distance mice covered while running. We found that female mice

154 in both cohorts ran more than male mice reaching up to $\sim 10 \mathrm{~km}$ per $24 \mathrm{hr}$. Male mice in cohort 2

155 displayed the lowest running activity of $\sim 3 \mathrm{~km}$ per day. On average female C57BI/6 mice

156 covered larger distances than male C57BI/6 mice (Fig. 3C, $p=0.0065$; Kruskal-Wallis; $\mathrm{n}=12$ ),

157 even though variability can be high.

We next investigated the effect of group housing on running patterns by housing three

159 same sex mice together in each cage. We observed that group-housed mice also ran

160 predominantly in bouts during the dark cycle (Fig. 4A, B). However, the temporal running

161 patterns were different in the group housing scenario when compared to the single housing

162 scenario. As a group, wheel locomotion increased in both female and male cages with a

163 temporal pattern that became more continuous, though bouts were often clearly visible (Fig. 4A,

164 B). Overall, group-housed mice maintained peak velocities of $\sim 80 \mathrm{~cm} / \mathrm{s}$, similar to single-housed

165 mice (Fig. 4C-F). The increased continuous running activity suggested a change in the long-

166 term (>100 s) temporal organization of how mice utilize the running wheel compared to when

167 they are placed in single housing conditions. Indeed, the autocorrelation in running activity

168 changed from an exponential distribution typical for single-housed runs (cf. Figs. 2D, 3B)

169 towards a power law (Fig. 4E, F). This power law suggests current running activity to have long-

170 lasting influence on running activity up to $500 \mathrm{~s}$ later.

171 We next compared the average distance run by mice when single-housed (Fig. 3C) to

172 the total running activity of the same mice when group-housed (Fig. 4G). We found that group- 
173 housed mice on average ran between 20 - $70 \%$ less compared to when they were single-

174 housed (Fig. 4H). We found this reduction in all groups, particularly prominent among female

175 mice, yet also in male mice that ran the least.

176

\section{Discussion}

178 The present study introduces a low-cost, scalable acquisition system to study voluntary wheel

179 running of mice in their respective cages. We analyzed the temporal organization of the

180 voluntary running of mice when having exclusive access to a wheel (single-housed) compared

181 to when mice shared a wheel with up to 2 others of the same sex (group-housed). We found

182 that mice on average ran up to $70 \%$ less when group-housed and their overall running patterns

183 change from isolated running bouts at maximum speed to more continuous running activity

184 suggesting potential social interactions. These findings suggest that access to a running wheel

185 provides an environmental enrichment for which mice compete for.

187 Comparison with alternative available cage wheel recording systems

188 We used a standard NI data acquisition board and added simple external magnetic switch

189 circuits. The design was sturdy, yet flexible enough to rapidly record mice in their home cage

190 over extended periods of time. Detailed protocols have been published for studying voluntary

191 wheel running in mice (Goh and Ladiges, 2015) and numerous locomotion monitoring systems

192 have been developed for mice based on various techniques such as horizontal, rotating

193 platforms (Zhu et al., 2021), and video tracking (Kobayashi et al., 2020) including tagging

194 individual mice with RFID tags (for review see (Bains et al., 2018)). Our low-cost monitoring

195 system, in contrast to other commercially available systems that use their own caging system,

196 can be easily implemented and flexibly employed in many animal facilities and laboratory

197 environments. Our system uses magnetic switches which, in comparison to optical detectors, 
are resistant to dust. Moreover, since we utilized four magnets per wheel, we could track wheel

199 motion with high precision allowing the detailed analysis of running bouts.

201 Single- vs. group-housed running

202 The present study shows that single-housed mice easily run $>10 \mathrm{~km} /$ day, yet some male mice

203 run significantly less indicating a high level of variability even in inbred strains. Therefore,

204 studies of running behavior benefit from low-cost monitoring systems. The finding that group-

205 housed mice barely run a longer distance together per day suggests a significant limitation in

206 wheel access during group housing for individual mice. The consequences of these changes

207 and limitations during social housing are not well understood yet. Specifically, it is not clear how

208 mice divide this limited resource during group housing. The reduction in overall running during

209 group housing was also found in male mice that ran the least amount (Cohort 2) suggesting that

210 this reduction does not solely arise from limited occupancy capacity of the wheel when mice are

211 group-housed.

We found an exponential decay for the temporal organization of running bouts during

213 single housing that changed to a power law decay during group housing. Such a profound

214 change in autocorrelation suggests potential long-term, social interactions among mice when

215 sharing a single wheel. The distribution in the duration of locomotion and resting times have

216 been successfully described by exponential and power law functions respectively in both human

217 and rodents (Anteneodo and Chialvo, 2009; Nakamura et al., 2007). Those functional

218 differences suggest different mechanisms underlying running and resting. Indeed, changes in

219 the power law slope have been linked to mental disease in humans (Chapman et al., 2017;

220 Nakamura et al., 2007). Identifying periods of minimal locomotion requires a thorough

221 exploration of their threshold dependency. In addition, the animal producing the current running

222 bout needs to be identified during group housing. These technical challenges are beyond the

223 scope of the current work. Instead, we used the robust measure of the autocorrelation, which 
224 does not require the introduction of a threshold and is sensitive to both changes in running

225 duration and quiet periods between running bouts. Our demonstration of a significant change in

226 autocorrelation between single and group housing supports further exploration of the underlying

227 temporal organization in running activity. For example, simultaneous video recordings along

228 with remote readable ID-tagging could be used to study the social hierarchy established during

229 wheel running under those conditions. If socially stratified, running wheel occupancy under

230 social housing conditions could be used as a proxy to identify social hierarchy among colonies.

231 This method might be expandable to transgenic strains, which demonstrate distinct locomotion

232 activity patterns in response to their LD-cycle (Fuochi et al., 2021; Kopp, 2001). Differences in

233 locomotion activity patterns and/or wheel occupancy could be used to identify differences in

234 social hierarchies or social communication in models of disease, changes in social hierarchies

235 in response to specific drug treatments (Kobayashi et al., 2020). 


\section{Figures}

\section{Figure 1}

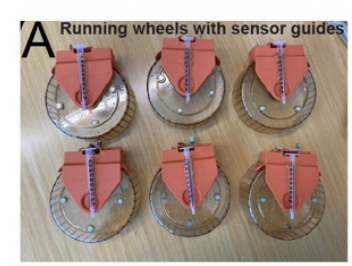

D Nidatáa acquisition board
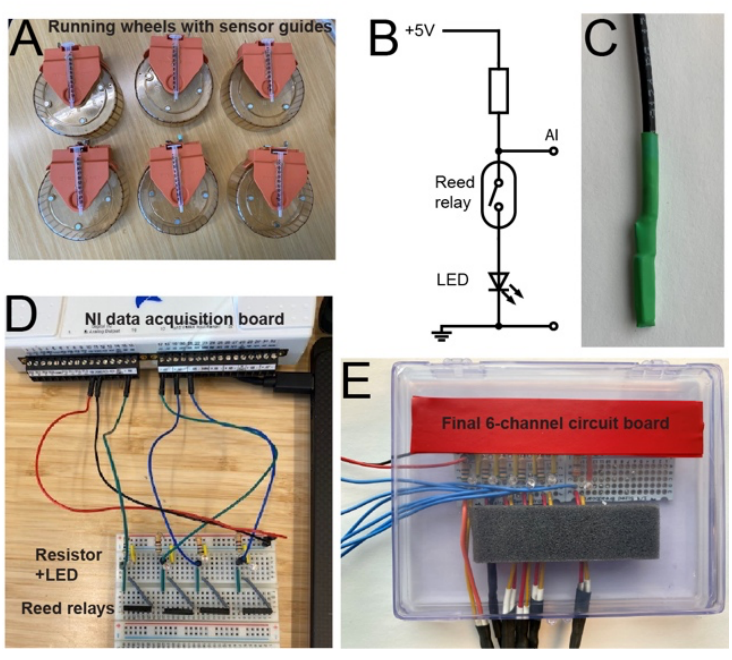

Figure 1. Low-cost data acquisition system to assess spontaneous wheel running

\section{0 (locomotion) in mice.}

241 (A) Plastic running wheels (Tecniplast, USA) modified for low-cost data acquisition by attaching

242 round neodymium magnets $(6 \times 3 \mathrm{~mm})$ and a $1 \mathrm{ml}$ outer syringe tubing to hold the magnetic

243 induction switch. The syringe tube is positioned such that the collar prevents mice from

244 accessing the sensor cable. (B) Circuit layout connects multiple channels with magnetic

245 induction switches (Reed) to the National Instruments (NI) data acquisition board (USB-6215).

246 Four channels and +5 V, DC (red), ground (black) for power support are shown. (C) Channel

247 circuit diagram. (D) Compact 6-channel circuit board with attached sensor cables (expandable

248 to 16 channels). 


\section{Figure 2}
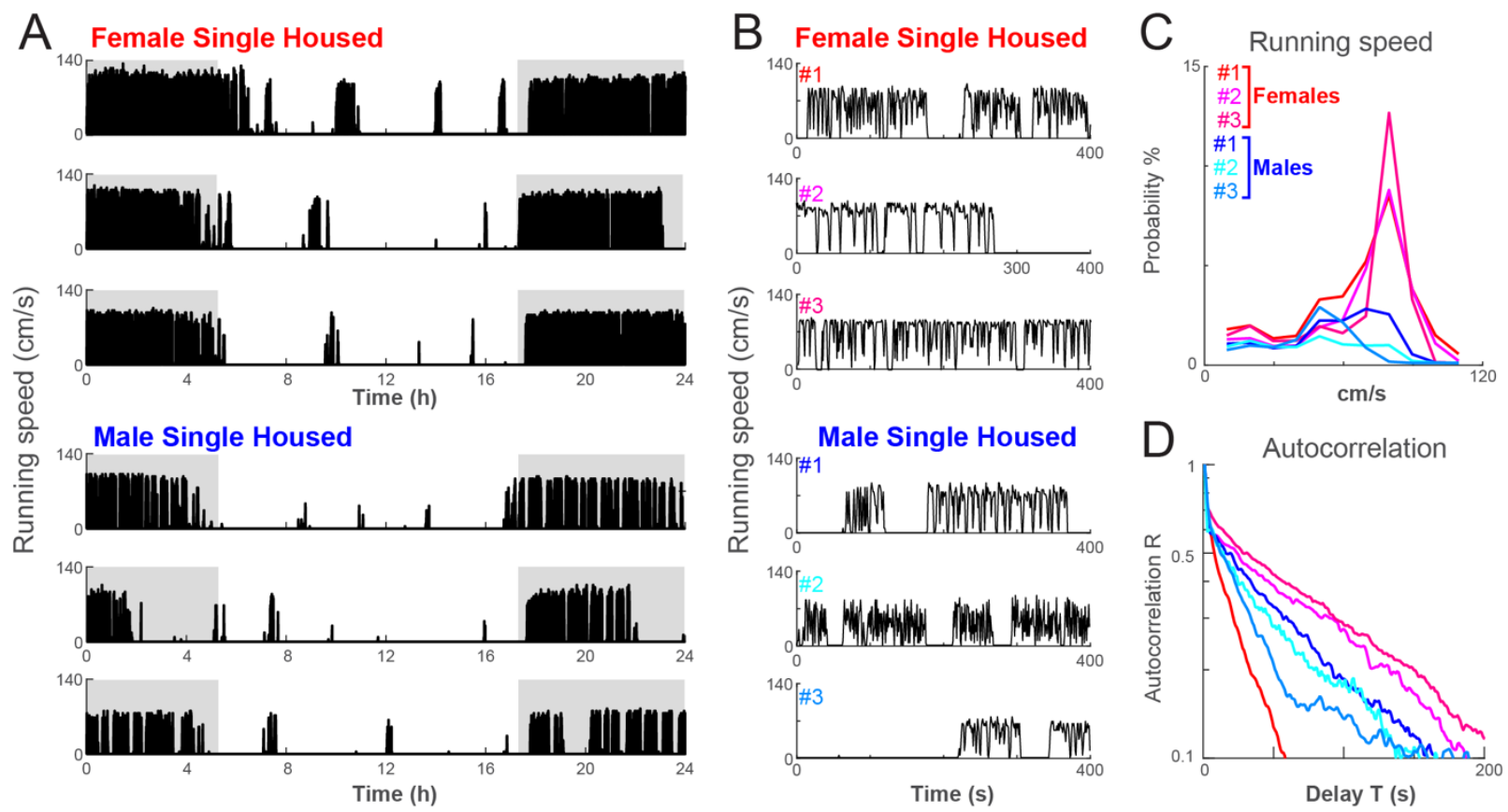

Figure 2. Wheel running activity in C57BI/6 mice exhibits individual running patterns and sex differences.

253 (A) Mice run predominantly during the D-phase at speeds that can reach up to $120 \mathrm{~cm} / \mathrm{s}$. During 254 the L-phase, intermittent running bouts are observed which are not synchronized between mice 255 (Cohort 2). Grey: D-phase. Simultaneous recording of $n=6$ mice housed individually for one 24 256 h LD-cycle. (B) Wheel running of individual mice at higher temporal resolution. Single mice run 257 in 'bouts', periods of rapid running that terminate abruptly. Note that some mice maintain 258 consistently high running speed during bouts (row 2, 3), whereas other mice show large 259 fluctuations in speed during bouts (row 1, 5). (C) Corresponding speed distributions over a full 260 LD-cycle for single mice (for color code see $B$ ). (D) The exponential decay of the autocorrelation 261 in running activity (semi logarithmic plot) indicates independence in the temporal occurrence of 262 running bouts for single-housed mice (for color code see $B$ ). 


\section{Figure 3}
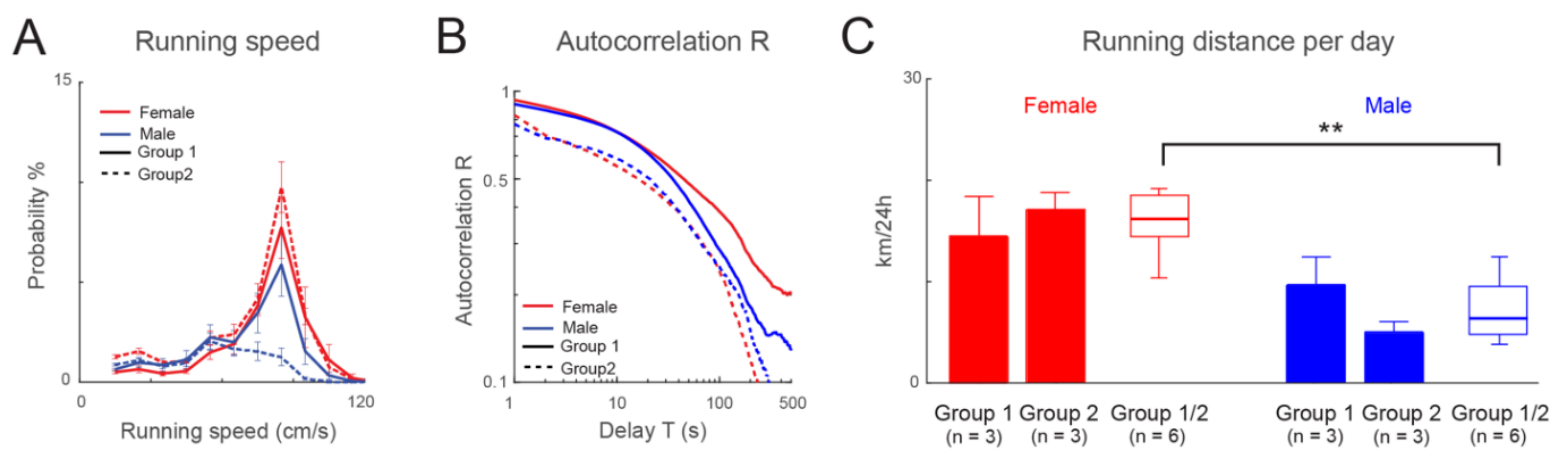

265 Figure 3. Female C57BI/6 mice run further than male C57BI/6 mice

266 (A) Summary in speed distributions for mice during single housing separated by cohort and sex.

267 Females displayed similar distributions for each cohort. Running activity of male mice in cohort

2681 was similar to that of female mice, whereas male mice of cohort 2 ran less. (B) Corresponding

269 average decay in time course for single-housed mice separated by cohort and sex. In loglog

270 coordinates, the exponential decay appears as a downward curved function. (C) Summary

271 statistics for total distance run in single-housed mice (number in brackets indicate number of

272 animals recorded). Note that females tend to run longer distances per day in both cohorts.

273 Number in brackets indicate animals tested. All data are mean \pm SD. 


\section{Figure 4}

A

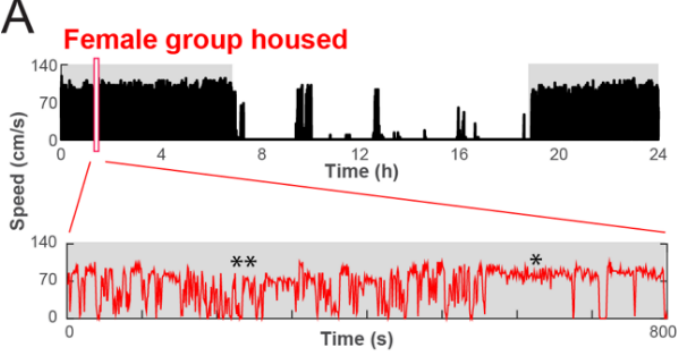

B

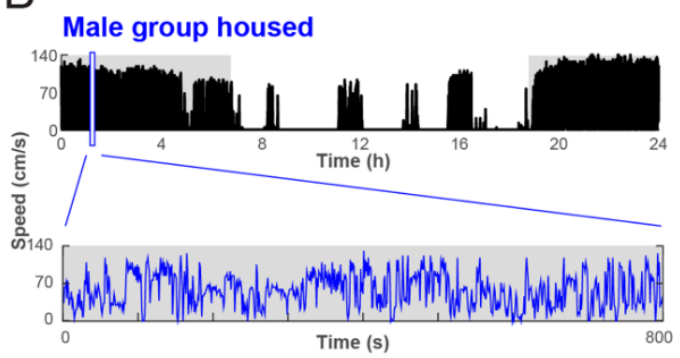

C Running speed (Single Day)

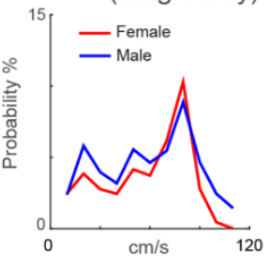

Autocorrelation (Single Day)

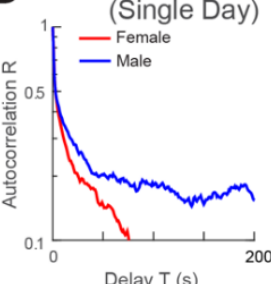

Delay T (s)

G Running distance/day

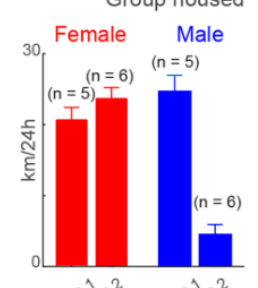

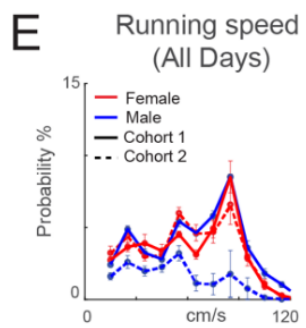

F Autocorrelation

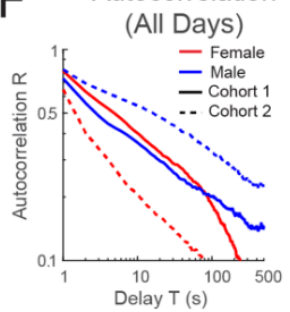

H Distance Reduction Group vs Single housed

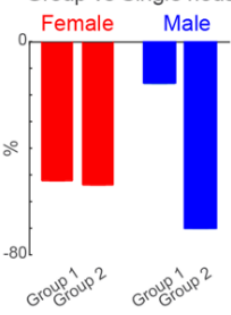

Figure 4. Group-housing reduces total distance in voluntary wheel running when compared to single-housing.

279 (A) Group-housed C57BI/6 mice ( $\mathrm{n}=3$ mice per cage) run predominantly during the D-phase.

During the L-phase, intermittent running bouts are observed which are not synchronized between cages. Grey: D-phase. Simultaneous recording of mice housed in groups of $n=3$ separated by sex for a 24 h LD-cycle. (B) Enlarged temporal time course from $A$ identifies segments of highly-variable running speed in male and female cages. Highly variable, lowspeed epochs $\left({ }^{* *}\right)$ can alternate with stable, high-speed epochs $\left({ }^{*}\right)$ as found for single-housed mice. (C). Similar speed distribution in group-housed male and female mice (Cohort 1; single day). (D) Summary in speed distribution for the two cohorts separated by group-housed males 
287 and females ( $n=5$ days for cohort $1 ; n=6$ days for cohort 2). (E) Slow decay in the

288 autocorrelation for female and male cages reflects the continuous, long-term interactions

289 between animals (semi-log plot; cohort 1; single day). (F) Power law decay in the

290 autocorrelation of running activity visible as linear relationship in loglog plot. This power law

291 relationship uncovers long-term temporal correlations during group housing (all cohorts; cf.

292 exponential decay for single-housing in Fig. 2D, 3B). (G) Summary statistics for total distance

293 run per day during group housing (number in brackets indicate days recorded). (H) Reduction

$294(\mathrm{R})$ in running distance in group housing. All mice experience a reduction in total distance run 295 per day during group housing of up to $70 \%$. 


\section{References}

Anteneodo, C., and Chialvo, D.R. (2009). Unraveling the fluctuations of animal motor activity. Chaos 19, 033123.

Bains, R.S., Wells, S., Sillito, R.R., Armstrong, J.D., Cater, H.L., Banks, G., and Nolan, P.M. (2018). Assessing mouse behaviour throughout the light/dark cycle using automated in-cage analysis tools. J. Neurosci. Methods 300, 37-47. https://doi.org/10.1016/j.jneumeth.2017.04.014. brain plasticity: impact of environmental enrichment. Cell Death Differ. 17, 1092-1103. 10.1038/cdd.2009.193.

307 Chapman, J.J., Roberts, J.A., Nguyen, V.T., and Breakspear, M. (2017). Quantification of free308 living activity patterns using accelerometry in adults with mental illness. Scientific Reports 7 , 309 43174. 10.1038/srep43174.

310 Elias, P.K., Elias, M.F., and Eleftheriou, B.E. (1975). Emotionality, exploratory behavior, and 311 locomotion in aging inbred strains of mice. Gerontology 21, 46-55.

312 Fuochi, S., Rigamonti, M., lannello, F., Raspa, M., Scavizzi, F., de Girolamo, P., and D’Angelo, 313 L. (2021). Phenotyping spontaneous locomotor activity in inbred and outbred mouse strains by 314 using Digital Ventilated Cages. Lab Animal 50, 215-223. 10.1038/s41684-021-00793-0.

315 Goh, J., and Ladiges, W. (2015). Voluntary wheel running in mice. Current protocols in mouse 316 biology 5, 283-290.

317 lannello, F. (2019). Non-intrusive high throughput automated data collection from the home 318 cage. Heliyon 5, e01454. https://doi.org/10.1016/j.heliyon.2019.e01454.

319 Kempermann, G. (2019). Environmental enrichment, new neurons and the neurobiology of 320 individuality. Nature Reviews Neuroscience 20, 235-245. 10.1038/s41583-019-0120-x.

321 Kobayashi, K., Shimizu, N., Matsushita, S., and Murata, T. (2020). The assessment of mouse 322 spontaneous locomotor activity using motion picture. Journal of Pharmacological Sciences 143, 323 83-88. https://doi.org/10.1016/j.jphs.2020.02.003.

324 Kopp, C. (2001). Locomotor activity rhythm in inbred strains of mice: implications for behavioural 325 studies. Behav. Brain Res. 125, 93-96.

326 Koteja, P., Swallow, J.G., Carter, P.A., and Garland, T., Jr. (1999). Energy cost of wheel running in house mice: implications for coadaptation of locomotion and energy budgets. Physiol Biochem Zool 72, 238-249. 10.1086/316653.

329 Nakamura, T., Kiyono, K., Yoshiuchi, K., Nakahara, R., Struzik, Z.R., and Yamamoto, Y. (2007). 330 Universal scaling law in human behavioral organization. Phys. Rev. Lett. 99, 138103.

331 Pasquarelli, N., Voehringer, P., Henke, J., and Ferger, B. (2017). Effect of a change in housing conditions on body weight, behavior and brain neurotransmitters in male C57BL/6J mice. Behav. Brain Res. 333, 35-42.

334 Rhodes, J.S., Garland Jr, T., and Gammie, S.C. (2003). Patterns of Brain Activity Associated With Variation in Voluntary Wheel-Running Behavior. Behav. Neurosci. 117, 1243-1256.

337 Roberts, M.D., Gilpin, L., Parker, K.E., Childs, T.E., Will, M.J., and Booth, F.W. (2012).

338 Dopamine D1 receptor modulation in nucleus accumbens lowers voluntary wheel running in rats 
339 bred to run high distances. Physiol. Behav. 105, 661-668.

340 https://doi.org/10.1016/j.physbeh.2011.09.024.

341 Sherwin, C.M. (1998). The use and perceived importance of three resources which provide 342 caged laboratory mice the opportunity for extended locomotion. Appl. Anim. Behav. Sci. 55,

343 353-367. https://doi.org/10.1016/S0168-1591(97)00049-X.

344 Stryker, M.P., and Löwel, S. (2018). Amblyopia: New molecular/pharmacological and 345 environmental approaches. Vis. Neurosci. 35.

346 Zhu, M., Kasaragod, D.K., Kikutani, K., Taguchi, K., and Aizawa, H. (2021). A Novel

347 Microcontroller-Based System for the Wheel-Running Activity in Mice. eneuro 8,

348 ENEURO.0260-0221.2021. 10.1523/ENEURO.0260-21.2021.

349 Zhu, X., Ottenheimer, D., and DiLeone, R.J. (2016). Activity of D1/2 receptor expressing 350 neurons in the nucleus accumbens regulates running, locomotion, and food intake. Frontiers in 351 behavioral neuroscience 10, 66 . 